\title{
Thinking Beyond HLH: Clinical Features of Patients with Concurrent Presentation of Hemophagocytic Lymphohistiocytosis and Thrombotic Microangiopathy
}

\author{
Nicholas J. Gloude ${ }^{1,2}$. Christopher E. Dandoy ${ }^{3,4}$ - Stella M. Davies ${ }^{3,4} \cdot$ Kasiani C. Myers ${ }^{3,4} \cdot$ Michael B. Jordan $^{3,4}$. \\ Rebecca A. Marsh ${ }^{3,4}$ - Ashish Kumar ${ }^{2,3}$. Jack Bleesing ${ }^{3,4}$ - Ashley Teusink-Cross ${ }^{4,5}$. Sonata Jodele ${ }^{3,4}$
}

Received: 27 February 2020 / Accepted: 4 May 2020 / Published online: 23 May 2020

(C) Springer Science+Business Media, LLC, part of Springer Nature 2020

\begin{abstract}
Hemophagocytic lymphohistiocytosis (HLH) is a syndrome of excessive immune system activation driven mainly by high levels of interferon gamma. The clinical presentation of HLH can have considerable overlap with other inflammatory conditions. We present a cohort of patients with therapy refractory HLH referred to our center who were found to have a simultaneous presentation of complement-mediated thrombotic microangiopathy (TMA). Twenty-three patients had therapy refractory HLH (13 primary, 4 EVB-HLH, 6 HLH without known trigger). Sixteen (69.6\%) met high-risk TMA criteria. Renal failure requiring renal replacement therapy, severe hypertension, serositis, and gastrointestinal bleeding were documented only in patients with HLH who had concomitant complement-mediated TMA. Patients with HLH and without TMA required ventilator support mainly due to CNS symptoms, while those with HLH and TMA had respiratory failure predominantly associated with pulmonary hypertension, a known presentation of pulmonary TMA. Ten patients received eculizumab for complement-mediated TMA management while being treated for HLH. All patients who received the complement blocker eculizumab in addition to the interferon gamma blocker emapalumab had complete resolution of their TMA and survived. Our observations suggest coactivation of both interferon and complement pathways as a potential culprit in the evolution of thrombotic microangiopathy in patients with inflammatory disorders like refractory HLH and may offer novel therapeutic approaches for these critically ill patients. TMA should be considered in children with HLH and multi-organ failure, as an early institution of a brief course of complement blocking therapy in addition to HLH-targeted therapy may improve clinical outcomes in these patients.
\end{abstract}

Keywords Hemophagocytic lymphohistiocytosis · Thrombotic microangiopathy · Complement · Interferon gamma . Eculizumab · Emapalumab

Electronic supplementary material The online version of this article (https://doi.org/10.1007/s10875-020-00789-4) contains supplementary material, which is available to authorized users.

Sonata Jodele

Sonata.jodele@cchmc.org

1 Department of Pediatrics, University of California San Diego, San Diego, USA

2 Division of Hematology Oncology, Rady Children's Hospital, San Diego, USA

3 Department of Pediatrics, University of Cincinnati College of Medicine, Cincinnati, USA

4 Division of Bone Marrow Transplantation and Immune Deficiency, Cincinnati Children's Hospital Medical Center, Cincinnati, USA

5 Division of Pharmacy, Cincinnati Children's Hospital Medical Center, Cincinnati, USA

\section{Introduction}

Hemophagocytic lymphohistiocytosis (HLH) is a rare clinical syndrome of excessive immune activation, characterized by signs and symptoms of extreme inflammation, driven mainly by interferon gamma (IFN $\gamma$ ) and other pro-inflammatory cytokines. Our understanding of HLH pathogenesis has significantly evolved in the past two decades. Though there are varied molecular etiologies, genetic HLH disorders most often develop due to either defective lymphocyte cytotoxicity or abnormal inflammasome activity. Defects in lymphocyte cytotoxicity due to perforin deficiency or degranulation defects lead to prolonged synapse times, increased production of inflammatory cytokines, and abnormal reciprocal activation of mononuclear phagocytes and cytotoxic NK cells and $\mathrm{T}$ cells. 
Activated mononuclear phagocytes promote hemophagocytosis and release of HLH biomarkers like ferritin, CXCL9, and IL-18BP. SAP deficiency uniquely impairs restimulation-induced cell death and, like CD27 and CD70 deficiency, prevents normal killing of EBV-infected B cells. The absence of XIAP permits pathogenic NLRP3 inflammasome activity and abnormal lymphocyte apoptosis, while activating mutations in NLRC4 drive pathologic NLRC4 inflammasome activity [1-6].

The current diagnostic criteria for HLH include either a genetic HLH diagnosis or five of the following eight clinical and/or laboratory criteria: fever, splenomegaly, cytopenias, hypertriglyceridemia and/or hypofibrinogenemia, hemophagocytosis, low or absent NK cell activity, elevated ferritin, and elevated sIL-2 receptor (sIL2R) (Table 1) [7]. The most commonly affected organs are the liver, spleen, central nervous system (CNS), and bone marrow. HLH is a syndrome with a variable clinical presentation, requiring comprehensive clinical evaluation to rule out other disorders and clinical syndromes, as clinical diagnostic criteria are not specific and there is considerable overlap with other conditions [8-11]. We present a group of patients referred to our center for the management of HLH, who also had a simultaneous presentation of clinically significant thrombotic microangiopathy (TMA). TMA is an increasingly recognized clinical syndrome that results from vascular endothelial injury and may occur in the setting of excessive inflammation such as that seen following a toxic injury (spider or snake bite, radiation, or chemotherapy), in highly inflammatory diseases like systemic lupus erythematosus (SLE), and after stem cell transplantation (TATMA) or solid organ transplant [12]. TMA presents with a constellation of laboratory features and a specific pattern of organ injury [13]. TMA diagnostic criteria include either a histologic diagnosis on tissue biopsy of microangiopathic changes or at least five of the seven laboratory and clinical markers listed in Table 1. If not recognized, TMA can progress to multi-organ failure. Complement system dysregulation is a known pathogenic pathway leading to TMA, and complement blocking agents are used therapeutically with some success [14]. However, other inflammatory pathways may contribute to the endothelial injury that initiate and sustain TMA, and can likely be targeted to improve clinical outcomes further $[15,16]$.

Our observations suggest that co-activation of both interferon and complement pathways is important in the evolution of TMA and offers novel therapeutic approaches for these critically ill patients.

\section{Methods}

\section{Study Subjects}

Consecutive patients of any age with hemophagocytic lymphohistiocytosis (HLH) refractory to first-line therapy who were treated at our institution from January 2012 through December 2018 were identified by retrospective chart review after Institutional Review Board (IRB) approval. All patients were prospectively screened for TMA as part of clinical care as previously described [17]. Patient demographics, disease characteristics, HLH and TMA-targeted therapy,

Table 1 Diagnostic criteria for HLH and TMA

Hemophagocytic lymphohistiocytosis (HLH)

A. Genetic HLH diagnosis

or

B. Five of the eight criteria met

1. Fever $\geq 38.5^{\circ} \mathrm{C}$

2. Splenomegaly

3. Cytopenias (affecting at least 2 cells lines)

-Hemoglobin $<9 \mathrm{~g} /$ dL-Platelets $<100 \times 10^{9} /$ L-Neutrophils $<1 \times 10^{9} / \mathrm{L}$

4. Hypertriglyceridemia and/or hypofibrinogenemia $(<150 \mathrm{mg} / \mathrm{dL})$

5. Hemophagocytosis

6. Low or absent NK cell activity

7. Elevated ferritin $>500 \mathrm{ng} / \mathrm{mL}$

8. Elevated sIL-2 receptor (sIL2R) $>2400 \mathrm{U} / \mathrm{mL}$ or elevated above defined normal laboratory value

Thrombotic microangiopathy (TMA)

A. Tissue diagnosis

B. Five of the seven criteria met

1. $\mathrm{LDH}$ above normal value for age

2. Schistocytes on peripheral blood smear

3. De novo thrombocytopenia or require platelet transfusions

4. De novo anemia or require $\mathrm{RBC}$ transfusions

5. Hypertension $>99 \%$ for age ( $<18$ years of age) or $140 / 90$ ( $\geq 18$ years of age) or receiving antihypertensive therapy

6. Proteinuria $\geq 30 \mathrm{mg} / \mathrm{dL}$ on random urinalysis $\times 2$ or random urine protein creatinine ratio $\geq 2 \mathrm{mg} / \mathrm{mg}$

7. Terminal complement activation: elevated plasma sC5b-9 above normal limit of $\geq 244 \mathrm{ng} / \mathrm{mL}$, or elevated above defined normal laboratory value Note: 6 and 7 are high-risk TMA features 
complications, intervention, and laboratory assessment were captured from the electronic medical record.

\section{Diagnostic Testing}

HLH and TMA diagnoses were made prospectively using published diagnostic criteria (Table 1) [7, 17]. Laboratory tests were performed as part of routine clinical care in a CLIAcertified laboratory. Routine institutional TMA monitoring included daily complete blood count (CBC) with attention to schistocytes; twice a week lactate dehydrogenase (LDH); and weekly haptoglobin, free plasma hemoglobin, and urinalysis with a random urine protein/creatinine ratio. For subjects < 18 years of age, hypertension was defined as a blood pressure $>99 \%$ for age, sex, and height [18]. Hypertension for subjects $\geq 18$ years of age was defined as a blood pressure $\geq 140$ / $90 \mathrm{mmHg}$. The number of antihypertensive medications, not including diuretics, required to maintain the blood pressure below the targeted hypertension threshold (99th centile for age) were counted for each patient, and hypertension was classified as severe if management included $>2$ antihypertensive medications or a continuous antihypertensive medication infusion for $>12 \mathrm{~h}$.

Terminal complement activation was tested by measuring the level of sC5b-9 in blood and was checked in patients with elevated LDH and nephrotic range proteinuria (random urine protein/creatinine ratio $\geq 2 \mathrm{mg} / \mathrm{mg}$ ). sC5b-9 was reported as elevated if the measurement in the blood was $>244 \mathrm{ng} / \mathrm{mL}$ by enzyme immunoassay (EIA).

Patients with hematologic signs of TMA who had both nephrotic range proteinuria and complement activation as measured by elevated sC5b-9, or evidence of multi-organ injury syndrome (MODS), were classified as high-risk TMA and were offered therapy with the terminal complement blocker, eculizumab. Eculizumab dosing was based on drug pharmacokinetics and pharmacodynamics (PK/PD) as previously published $[17,19]$. All patients on eculizumab therapy received antimicrobial prophylaxis against meningococcus until the drug was cleared after therapy completion and complement function was restored.

MODS was diagnosed when a patient had symptoms of HLH/TMA and dysfunction of two or more organ systems: renal failure requiring renal replacement therapy (RRT) or cystatin $\mathrm{C}$ glomerular filtration rate $(\mathrm{GFR})<50 \mathrm{~mL} / \mathrm{min}$, invasive or non-invasive positive pressure ventilator support for $>24 \mathrm{~h}$, diagnosis of pulmonary hypertension (as determined by echocardiogram and cardiology consultation), serositis (pleural or pericardial effusions), severe hypertension requiring either $\geq 2$ medications or continuous infusion of an antihypertensive for $>12 \mathrm{~h}$ to maintain blood pressure $<99 \%$ for age, CNS symptoms (seizures, bleeding, posterior reversible encephalopathy syndrome (PRES), or altered mental status), or gastrointestinal symptoms (ileus and/or bleeding) [20-24].

\section{Results}

Twenty-three patients with frontline therapy refractory HLH were treated at our institution during the study period. All patients met clinical HLH criteria, as listed in Table 1. Twelve (52\%) had confirmed genetic HLH, 4 (17.4\%) had EBV-associated secondary HLH, and 7 (30.4\%) met HLH clinical diagnostic criteria but did not have any identified genetic abnormality, nor EBV infection. Sixteen of 23 patients (70\%) had CNS symptomatology such as altered mental status, encephalopathy, or seizures, and seven of these patients also had a documented CNS bleed, as well as radiographic evidence of posterior reversible encephalopathy syndrome (PRES). All patients were initially treated with etoposide and dexamethasone except for one patient who received cytarabine and methylprednisolone. All patients had either disease progression on therapy or had an HLH disease relapse after achieving brief remission with additional agents and high dose dexamethasone ( $\geq 10 \mathrm{mg} / \mathrm{m}^{2}$ ) (Supplemental Table 1A). Two patients had HLH relapse after allogenic HCT. Seventeen patients received emapalumab a monoclonal antibody directed against interferon gamma (IFN $\gamma$ ), as a targeted HLH therapy for refractory disease [25]. Fourteen of them received emapalumab as part of the clinical study and three for compassionate use. All patients had active HLH signs including elevated sIL2R at the start of emapalumab.

\section{Patients with HLH and TMA $(n=16)$}

All patients initiated prospective monitoring for thrombotic microangiopathy (TMA) as part of clinical care on arrival to our institution as listed in "Methods" section. Sixteen of 23 patients $(70 \%)$ met clinical and laboratory criteria for highrisk TMA in addition to their diagnosis of HLH, including elevated lactate dehydrogenase (LDH), presence of schistocytes in the blood, transfusion-dependent Coombs negative hemolytic anemia, transfusion-dependent thrombocytopenia, nephrotic range proteinuria, and severe hypertension. High-risk TMA features included nephrotic range proteinuria and complement activation (Table 1) and/or multi-organ injury. Nine patients were tested for complement gene abnormalities using an institutional aHUS genetic susceptibility panel (C3, CFB, CFH, CFHR1, CFHR3, CFHR5, CFI, and MLPA analysis for $C H F R 1 / C H F R 3$ deletion). Six patients had at least one complement gene variant identified. Three subjects were heterozygous for CFHR3-CFHR1 by multiple ligationdependent probe amplification (MLPA) analysis that has been reported in heterozygous state in stem cell transplant 
recipients with TMA. One subject had $C F H R 3$ and $C F H R 1$ partial duplication, which is of uncertain clinical significance (VUCS). Three subjects had more than 1 gene variant identified, including a likely pathogenic variant in $C F I$ (c.1246A >C(p.I416L, het), CFI (c.1217G >A(p.R406H),het) variant reported in macular degeneration and CFHR5 (c.486_487insAA(p.E163fs) heterozygous for a frameshift mutation that has been associated with aHUS (Supplemental Table 1A, B).

Fourteen patients were tested for the evidence of terminal complement pathway activation by measuring blood sC5b-9 (or soluble membrane attack complex), and thirteen of them had elevated sC5b-9 level (sC5b-9 range was 262 to > $1890 \mathrm{ng} / \mathrm{mL}$, normal is $<244 \mathrm{ng} / \mathrm{mL}$ ). Thirteen patients had nephrotic range proteinuria (random urine protein/creatinine ratio $>2 \mathrm{mg} / \mathrm{mg}$ ). Fifteen patients had multi-organ dysfunction syndrome (MODS) with two or more organ systems involved, and 12 of them required intensive care support. Twelve patients required positive pressure ventilation for respiratory failure, and four of them had pulmonary hypertension. One patient required ECMO support who did not survive and was found on autopsy to have diffuse alveolar hemorrhage. This patient had CMV viremia that could be a possible trigger for inflammatory process. Eight patients had renal failure; seven received renal replacement therapy (RRT), and one patient's family elected not to proceed with RRT. Two patients had histologic evidence of TMA in kidney on autopsy (Supplemental Table 1A). Eight additional patients had a > $50 \%$ decline in cystatin C GFR from their pre-transplant baseline. All patients with TMA had severe hypertension requiring more than two antihypertensive medications or a continuous antihypertensive medication infusion to maintain blood pressure below 99th percentile for age. Six had clinically significant serositis requiring pericardial or pleural drain placement. Eleven patients in this group had CNS symptoms such as seizure or encephalopathy attributed to HLH diagnosis, but six of these patients also had a documented CNS bleed clinically attributed to PRES (Table 2).

Ten of 16 patients in the HLH plus TMA group received complement blockade with eculizumab as a targeted therapy for thrombotic microangiopathy using personalized PK/PD dosing (Fig. 1). All of these patients had documented activation of terminal complement (elevated sC5b9). Four of these 10 treated patients received eculizumab together with primary HLH therapy with corticosteroid with or without etoposide. None of these four patients proceeded to HSCT, and two died with active TMA. The other six patients received eculizumab after starting treatment with the IFN $\gamma$ blocker emapalumab for refractory HLH. One patient started eculizumab 8 days after initiation of emapalumab and had resolution of TMA. The patient did not proceed to HCST due to a lack of suitable donor and is alive and well for more than 3 years after therapy. The other five patients in this group who proceeded to HSCT after HLH-targeted therapy had active TMA at the start of hematopoietic stem cell transplantation (HSCT). They continued complement blockade with eculizumab during HSCT with successful resolution of their TMA. All patients were alive, with only one developing liver GVHD after HSCT. The median number of eculizumab doses given to patients with TMA was 9 (range 2-11). The median time to complete TMA resolution and organ function recovery was 16 weeks (9-21 weeks). Eculizumab therapy was well tolerated without any adverse events in this cohort.

Six patients with HLH and TMA did not receive eculizumab (Fig. 1). One patient had resolution of TMA with plasma exchange therapy before eculizumab was available and successfully underwent allogeneic HSCT. In two other patients, TMA resolved with emapalumab given for HLH. Of these two, one patient's family opted not to proceed to HSCT and one patient subsequently underwent HSCT. Two patients died shortly after transfer to our institution with both HLH and TMA after initiation of emapalumab, but prior to HSCT. One patient died prior to the use of either eculizumab or emapalumab.

\section{Patients With HLH and Without TMA $(n=7)$}

Seven of 23 patients ( $30 \%$ ) with HLH did not meet criteria for TMA and did not have evidence of complement activation in the blood (Fig. 1). Four of these seven patients required intensive care admission for respiratory support, mostly due to CNS symptoms such as status epilepticus. Five patients developed CNS symptoms, all attributed to HLH. One patient who was diagnosed with HLH 3 days after birth had evidence of a perinatal intraventricular bleed (IVH). None of the patients without TMA required renal replacement therapy, and none had severe hypertension. MODS was present in four of these patients.

\section{Discussion}

We observed a high incidence of clinically significant complement-mediated thrombotic microangiopathy (TMA) associated with multi-organ injury in children and young adults with a diagnosis of HLH. TMA has not been previously associated with interferon gamma-driven diseases like HLH [26], but TMA has been reported as an iatrogenic therapylimiting side effect in patients receiving interferon alpha and beta therapy [27-31]. We hypothesized that high levels of interferon gamma in HLH might contribute directly to endothelial damage or injure endothelium through complement system activation. Our previous gene expression analysis in autologous stem cell recipients showed upregulation of interferon-responsive complement genes at the onset of TATMA that resolved after therapy with complement blocking 
Table 2 Demographic and disease characteristics

\begin{tabular}{|c|c|c|}
\hline & HLH with TMA 16/23 (70\%) & HLH without TMA 7/23 (30\%) \\
\hline Median age in years (range) & $1.5(0.4-23)$ & $1(0.1-4)$ \\
\hline Female gender & 10 & 4 \\
\hline Genetic HLH diagnosis & 7 & 5 \\
\hline No genetic diagnosis/no other triggers & 6 & 1 \\
\hline EBV-HLH (no genetic diagnosis) & 3 & 1 \\
\hline \multicolumn{3}{|c|}{ TMA diagnosis (requires 5 of 7 criteria to be met) } \\
\hline & $11 / 16$ met 7 criteria & $1 / 7$ met 4 criteria \\
\hline & $4 / 16$ met 6 criteria & $2 / 7$ met 3 criteria \\
\hline & $1 / 16$ met 5 criteria & $4 / 7$ met 2 criteria \\
\hline \multicolumn{3}{|l|}{ Organ injury } \\
\hline $\begin{array}{l}\text { Multi-organ dysfunction syndrome } \\
\text { (MODS) }\end{array}$ & $15 / 16(94 \%)$ & $4 / 7(57 \%)$ \\
\hline Hypertension, severe & $16 / 16(100 \%)$ & $0 / 7(0 \%)$ \\
\hline Nephrotic range proteinuria & $13 / 16(81 \%)$ & $1 / 7(14 \%)$ \\
\hline Elevated sC5b-9 (>244 ng/mL) & $13 / 14(93 \%)$ & $0 / 4(0 \%)$ \\
\hline Renal failure & $8 / 16(50 \%)$ & $0(0 \%)$ \\
\hline Renal replacement therapy & $7 / 16(44 \%)$ & $0 / 7(0 \%)$ \\
\hline Cystatin C GFR $<50 \mathrm{~mL} / \mathrm{min}$ & $13 / 16(81 \%)$ & $2 / 6(33 \%)$ \\
\hline Positive pressure ventilation & $12 / 16(75 \%)$ & $4 / 7(57 \%)$ \\
\hline Pulmonary hypertension & $4 / 15(27 \%)$ & $1 / 7(14 \%)$ \\
\hline Serositis requiring surgical interventions & $6 / 16(38 \%)$ & $0(0 \%)$ \\
\hline GI symptoms (bleeding, ileus) & $7 / 16(44 \%)$ & $0 / 7(0 \%)$ \\
\hline CNS symptoms (any) ${ }^{\mathrm{a}}$ & $11 / 16(69 \%)$ & $5 / 7(71 \%)$ \\
\hline CNS bleeding & $6 / 11(55 \%)$ & $1 / 7(14 \%)^{\mathrm{b}}$ \\
\hline PICU admission & $12 / 16(75 \%)$ & $4 / 7(57 \%)$ \\
\hline Death & $6 / 16(37.5 \%)$ & $3 / 7(43 \%)$ \\
\hline
\end{tabular}

Additional information is listed in Supplemental Table 1

${ }^{\text {a }}$ Clinically attributed to HLH diagnosis

${ }^{\mathrm{b}}$ Perinatal intraventricular hemorrhage (IVH)

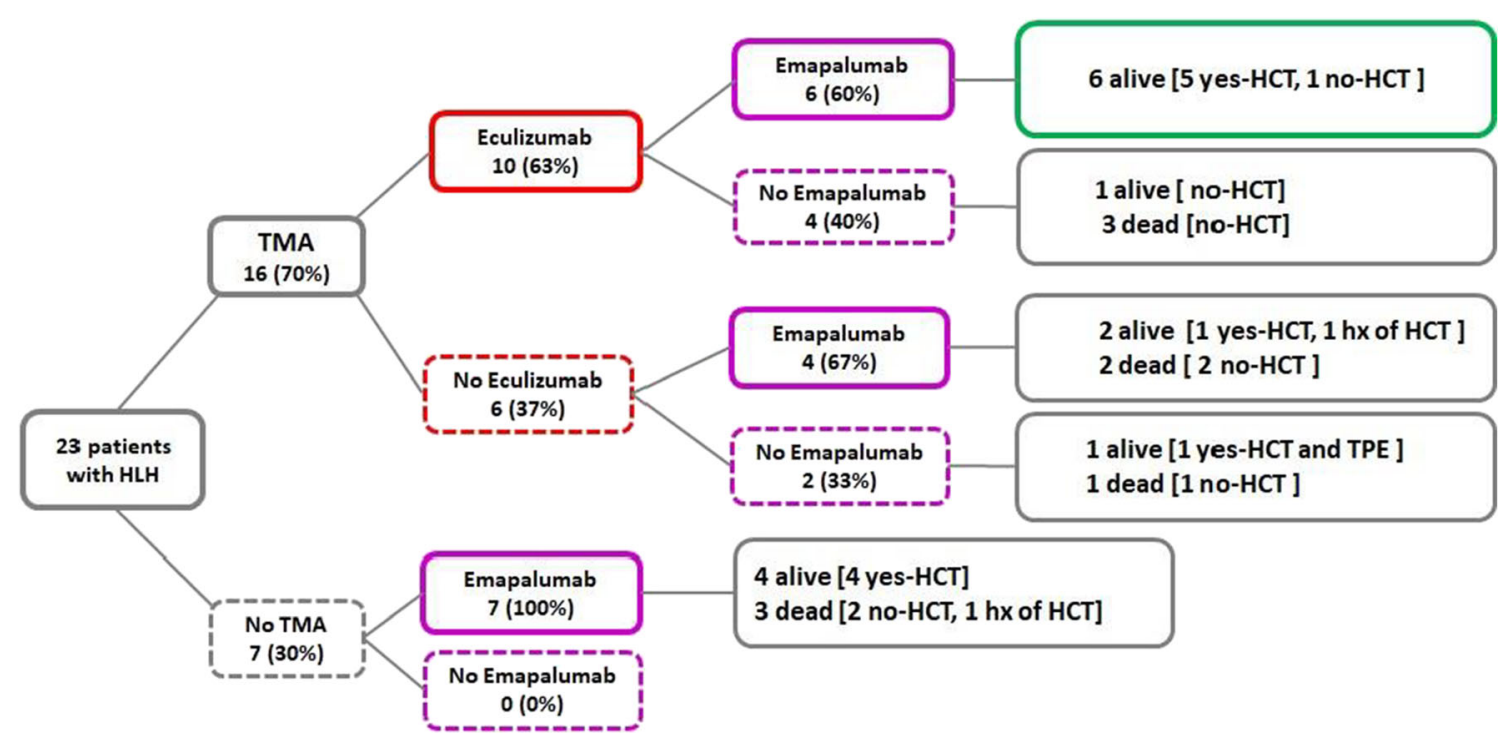

Fig. 1 Outcomes of patients with HLH based on treatment received 


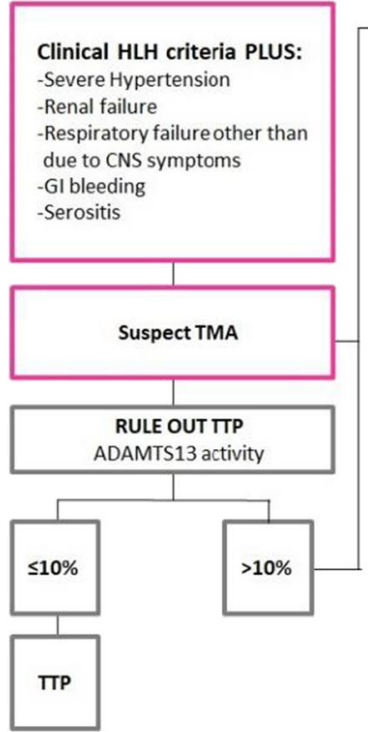

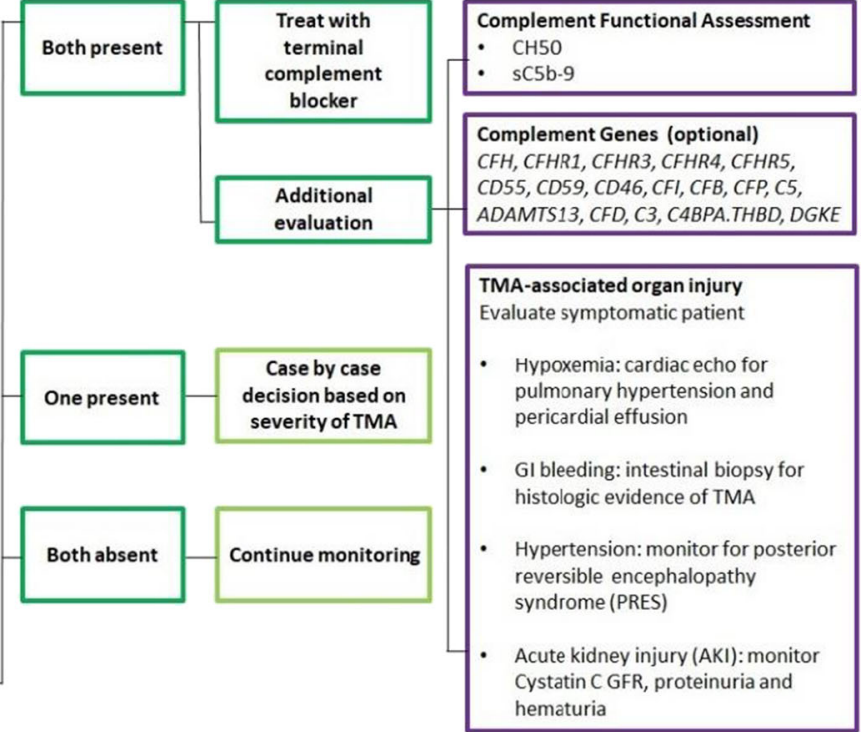

Fig. 2 TMA evaluation schema for patients with clinical diagnosis of HLH

agent eculizumab [32]. High levels of IFN $\gamma$ promote the formation of neutrophil extracellular traps (NETs), leading to complement activation via complement factor P (CFP) [33]. Terminal complement activation resulting in vascular endothelial injury is one of the important pathogenic pathways leading to high-risk TMA with multi-organ dysfunction syndrome. Uncontrolled terminal complement activation can maintain IFN $\gamma$ secretion via intracellular $\mathrm{C} 5 \mathrm{a}$ receptor production resulting in a cycle of continuing tissue injury [34]. Vascular endothelium injured by terminal complement can secrete interferons in addition to the robust production of IFN $\gamma$ by activated T cells, NK cells, and macrophages in patients with immune over-activation syndromes like HLH. While both interferons and complement are essential components of our immune response, over-activation of these pathways results in organ injury as "collateral damage" during high inflammatory states as seen in HLH and TMA.

In our prior prospective study examining complement genes in HSCT, we observed a significant association between TMA severity and outcomes in HLH patients undergoing HSCT who had complement gene variants identified. In this study, complement gene variants were more common in HLH subjects with TMA then those without TMA $(7 / 9,78 \%$ vs $0 / 8,0 \%, p=0.0023$ ). The median number of complement variants detected in these 7 HSCT recipients with HLH was 2 (range 1-5), and all patients with complement variants identified had severe TMA and multi-organ injury with an elevated blood sC5b-9 level indicating terminal complement over-activation. Transplant-related mortality was also significantly higher in HLH patients with TMA with six patients of the 9 patients dying from TMA-associated complications, while all HLH patients without TMA survived after transplant (6/9 $67 \%$ vs $0 / 8,0 \%, p=0.09$ ) [35]. Based on this prior observation, it is possible that patients with HLH who have complement gene variants in addition to the genetic defects of their primary disease may be more prone to rapid complement activation during active HLH, and may have more severe organ damage resulting from both interferon and complement co-injury of endothelial cells. Prompt control of overactivation of the interferon and complement systems is likely needed for successful resolution of both clinical conditions, HLH and TMA. Our study was limited by small sample size, so we were unable to perform any statistical analysis confirming benefit of eculizumab therapy in subjects with TMA. However, all patients with HLH and TMA who received the complement blocker eculizumab with the IFN $\gamma$ blocker emapalumab recovered and are doing well, supporting further investigation of this approach. Such targeted therapies can only be successfully applied if these co-existent hyperinflammatory conditions are appropriately recognized.

HLH often presents with liver failure and a hyperinflammatory syndrome mimicking sepsis that can ultimately lead to MODS due to coagulopathy, hypotension, and capillary leak. However, HLH rarely causes direct renal and pulmonary failure, or gastrointestinal bleeding in the absence of sepsis [36]. HLH commonly causes neutropenia, but the white blood cell counts are rarely affected in TMA. Patients presenting with a diagnosis of HLH and MODS (in particular renal failure, pulmonary failure, and uncontrolled hypertension that is more severe than expected with dexamethasone use) should be evaluated for co-existing TMA. These patients can benefit from evaluation for terminal complement activation by measuring sC5b-9 blood levels in addition to routine hematologic and renal TMA markers and organ function assessment (Fig. 2).

In our patient cohort, ventilator support in patients who had HLH but not TMA was generally required because of CNS symptoms, while patients with TMA were more likely to have 
Table 3 Organ injury at disease presentation

\begin{tabular}{|c|c|c|}
\hline Organ & Hemophagocytic lymphohistiocytosis (HLH) & Thrombotic microangiopthy (TMA) \\
\hline Liver & Transaminitis, coagulopathy, liver failure & Transaminitis, no coagulopathy \\
\hline CNS & $\begin{array}{l}\text { Seizures, encephalophagy due to CNS-HLH. Meningeal } \\
\text { enhancement, polymorphic white matter changes on MRI. } \\
\text { Elevated CSF protein and neopterin. }\end{array}$ & $\begin{array}{l}\text { Mental status changes. Seizures often associated with } \\
\text { hypertension. Evidence of PRES on brain MRI. CNS bleed in } \\
\text { non-coagulopathic patient. }\end{array}$ \\
\hline Kidney & $\begin{array}{l}\text { Primary injury is not common: secondary AKI due to medications, } \\
\text { capillary leak, hypotension }\end{array}$ & $\begin{array}{l}\text { AKI, nephrotic range proteinuria, renal failure, severe } \\
\text { hypertension. }\end{array}$ \\
\hline Heart & $\begin{array}{l}\text { Primary injury is not common, serositis due to third spacing, liver } \\
\text { failure }\end{array}$ & $\begin{array}{l}\text { Right heart failure as a result of pulmonary hypertension due to } \\
\text { pulmonary TMA. Pericardial effusion }\end{array}$ \\
\hline $\begin{array}{l}\text { Vascular } \\
\text { system }\end{array}$ & Hypotension mimicking sepsis due to capillary leak & $\begin{array}{l}\text { Hypertension more severe that can be attributed to } \\
\text { dexamethasone use }\end{array}$ \\
\hline Lungs & $\begin{array}{l}\text { Primary injury is not common, respiratory failure often related to } \\
\text { CNS symptomatology (airway protection), or capillary leak } \\
\text { ("wet lungs") }\end{array}$ & $\begin{array}{l}\text { Hypoxemia often with clear lungs. } \\
\text { Pulmonary hypertension, interstitial pulmonary bleeding leading } \\
\text { to ARDS }\end{array}$ \\
\hline GI & Primary injury is not common & Intestinal bleeding due to ischemic colitis \\
\hline Hemato-poietic & Neutropenia, anemia, thrombocytopenia & Anemia, thrombocytopenia, schistocytosis \\
\hline
\end{tabular}

$A K I$ acute kidney injury, PRES posterior reversable encephalopathy syndrome, ARDS acute respiratory distress syndrome

respiratory failure due to cardiorespiratory pathology, often pulmonary hypertension. HLH patients with hypoxemic acute respiratory failure should undergo diagnostic evaluation for pulmonary hypertension, a known presentation of TMA [21].

In our cohort of patients, renal failure associated with severe hypertension was exclusively seen in patients with HLH and TMA. While hypertension in a patient with HLH can be due to the use of dexamethasone, severe hypertension requiring multiple medications or a continuous infusion of antihypertensive medication should trigger evaluation for TMA. In contrast, the hyperinflammatory state seen in patients with HLH without TMA usually mimics sepsis and is more likely to present with hypotension. Nephrotic range proteinuria measured by random urine protein/creatinine ratio of $\geq 2 \mathrm{mg} / \mathrm{mg}$ in addition to severe hypertension should also prompt consideration of TMA in the differential diagnosis of patients with HLH and renal insufficiency. Patients with HLH are more likely to have fluid overload from liver failure leading to hepato-renal syndrome, whereas TMA primarily affects the renal vasculature resulting in direct kidney injury requiring renal replacement therapy, especially associated with hypertension.

CNS involvement in HLH is quite common at clinical presentation, and CNS bleeding can also occur, especially in thrombocytopenic patients with coagulopathy. CNS injury in HLH and TMA can be similar in clinical presentation, but certain features help distinguish CNS HLH, such as CSF pleocytosis, elevated CSF protein, and neopterin, along with radiologic abnormalities such as meningeal enhancement, polymorphic white matter changes, often with non-specific periventricular distribution. CNS injury in TMA most commonly occurs due to uncontrolled hypertension resulting in altered mental status, PRES, seizures, and/or CNS bleeding [37].

Intestinal bleeding has been reported in patients with HLH, but is not common, while TMA is known to cause bowel injury quite often resulting in severe abdominal pain and bleeding, as was seen in our study population $[24,38]$. If available, histologic tissue evaluation for TMA should be considered in patients with HLH and GI bleeding. Patients with clinically significant intestinal bleeding may require higher doses of therapeutic monoclonal antibodies due to increased drug clearance [22].

Our clinical observations suggest that HLH patients copresenting with complement-mediated TMA often suffer multi-organ injury and likely can benefit from a brief course of therapy with the complement blocking agent eculizumab while receiving therapy for HLH. Coadministration of both an interferon blocker and a complement blocker targeting both pathways in TMA might provide faster control. While complement activation in our patients with HLH and TMA was quite prominent, so PK/PD-based eculizumab dosing should be considered as drug clearance can be rapid, especially during a high inflammatory state. Based on our data, we anticipate that a brief course of eculizumab should be sufficient to control complement over-activation while HLH treatment is ongoing. Our patients with HLH and TMA received a median of six doses of eculizumab. Some of our patients started eculizumab late in disease presentation with severe organ injury and were not able to recover. Those who survived were able to discontinue eculizumab without recurrence of TMA successfully. Six patients who received both drugs, an interferon gamma blocker and a complement blocker, resolved both HLH and TMA, and are alive and well. 
Our study is limited by retrospective review of the data but provides preliminary insights into additional pathogenetic pathways of TMA that could be monitored and targeted, and brings awareness of the co-existence of HLH and TMA, especially in patients with multi-organ injury. While both HLH and TMA can have overlapping clinical presentation, they also have some distinctive clinical features and organ injury patterns that can be recognized using prospective monitoring (Table 3). Emerging new therapies like chimeric antigen receptor T cells (CART) cause cytokine release syndrome (CRS) due to high IFN $\gamma$ production, and disorders like systemic lupus erythematosus and Dego's syndrome present with high IFN $\alpha$ along with complement activation. The resolution of TMA without TMA-directed therapy has been observed in mild to moderate cases of TMA triggered by infection or engraftment after HSCT. However, severe TMA, especially presenting with significant complement activation, leads to high mortality due to multi-organ failure. Early institution of complement blocking therapy along with treatment of the primary disease may improve clinical outcomes in these patients.

\section{Conclusions}

Our observations suggest co-activation of both interferon and complement pathways as a potential culprit in the evolution of thrombotic microangiopathy in patients with inflammatory disorders like HLH and may offer novel therapeutic approaches for these critically ill patients. TMA should be considered in children with HLH and multi-organ failure, as an early institution of a brief course of complement blocking therapy in addition to HLH-targeted therapy may improve clinical outcomes in these patients.

\begin{abstract}
Acknowledgments Part of the research reported in this publication was supported by the Eunice Kennedy Shriver National Institute of Child Health and Human Development of the National Institute of Health (NIH) under award number R01HD093773 (S.Jodele). The content is solely the responsibility of the authors and does not necessarily represent the official views of the National Institutes of Health. Samples used in this study were collected and provided by the staff of the Cincinnati Children's Hospital Bone Marrow Tissue Repository who we thank for outstanding ongoing technical assistance. The authors would like to thank the clinical staff, patients, and families at Cincinnati Children's Hospital Medical Center.
\end{abstract}

Author Contributions NJG and SJ conceived the project, collected, analyzed, and interpreted the data, and wrote the manuscript. CED, SMD, $\mathrm{KCM}, \mathrm{MBJ}, \mathrm{RAM}, \mathrm{AK}, \mathrm{JB}$, and ATC analyzed and interpreted the data and edited the manuscript.

\section{Compliance with Ethical Standards}

Conflict of Interest NJG, CED, RAM, and JB have no interests to disclose. SJ and SMD have US patents pending. MBJ and AK are consultants for Sobi. ATC is on the speaker's bureau for Sobi. None of these funding sources had any input in the study design, analysis, manuscript preparation, or decision to submit for publication.

\section{References}

1. Buatois V, Chatel L, Cons L, Lory S, Richard F, Guilhot F, et al. Use of a mouse model to identify a blood biomarker for IFNgamma activity in pediatric secondary hemophagocytic lymphohistiocytosis. Transl Res. 2017;180:37-52 e32.

2. Prencipe G, Caiello I, Pascarella A, Grom AA, Bracaglia C, Chatel L, et al. Neutralization of IFN-gamma reverts clinical and laboratory features in a mouse model of macrophage activation syndrome. J Allergy Clin Immunol. 2018;141:1439-49.

3. Henter JI, Elinder G, Soder O, Hansson M, Andersson B, Andersson U. Hypercytokinemia in familial hemophagocytic lymphohistiocytosis. Blood. 1991;78:2918-22.

4. Xu XJ, Tang YM, Song H, Yang SL, Xu WQ, Zhao N, et al. Diagnostic accuracy of a specific cytokine pattern in hemophagocytic lymphohistiocytosis in children. J Pediatr. 2012;160:984-90 e981.

5. Bracaglia C, de Graaf K, Pires Marafon D, Guilhot F, Ferlin W, Prencipe G, et al. Elevated circulating levels of interferon-gamma and interferon-gamma-induced chemokines characterise patients with macrophage activation syndrome complicating systemic juvenile idiopathic arthritis. Ann Rheum Dis. 2017;76:166-72.

6. Lounder DT, Bin Q, de Min C, Jordan MB. Treatment of refractory hemophagocytic lymphohistiocytosis with emapalumab despite severe concurrent infections. Blood Adv. 2019;3:47-50.

7. Risma KA, Marsh RA. Hemophagocytic lymphohistiocytosis: clinical presentations and diagnosis. J Allergy Clin Immunol Pract. 2019;7:824-32.

8. Gurunathan A, Boucher AA, Mark M, Prus KM, O'Brien MM, Breese EH, et al. Limitations of HLH-2004 criteria in distinguishing malignancy-associated hemophagocytic lymphohistiocytosis. Pediatr Blood Cancer. 2018;65:e27400.

9. La Rosee P, Horne A, Hines M, von Bahr Greenwood T, Machowicz R, Berliner N, et al. Recommendations for the management of hemophagocytic lymphohistiocytosis in adults. Blood. 2019;133:2465-77.

10. Jordan MB, Allen CE, Greenberg J, Henry M, Hermiston ML, Kumar A, et al. Challenges in the diagnosis of hemophagocytic lymphohistiocytosis: recommendations from the North American Consortium for Histiocytosis (NACHO). Pediatr Blood Cancer. 2019;66:e27929.

11. Jordan MB, Allen CE, Weitzman S, Filipovich AH, McClain KL. How I treat hemophagocytic lymphohistiocytosis. Blood. 2011;118:4041-52.

12. Jodele S, Dandoy CE, Myers KC, El-Bietar J, Nelson A, Wallace $\mathrm{G}$, et al. New approaches in the diagnosis, pathophysiology, and treatment of pediatric hematopoietic stem cell transplantationassociated thrombotic microangiopathy. Transfus Apher Sci. 2016;54:181-90.

13. Jodele S, Laskin BL, Dandoy CE, Myers KC, El-Bietar J, Davies SM, et al. A new paradigm: diagnosis and management of HSCTassociated thrombotic microangiopathy as multi-system endothelial injury. Blood Rev. 2015;29(3):191-204.

14. Jodele S. Complement in pathophysiology and treatment of transplant-associated thrombotic microangiopathies. Semin Hematol. 2018;55:159-66.

15. Dvorak CC, Higham C, Shimano KA. Transplant-associated thrombotic microangiopathy in pediatric hematopoietic cell transplant recipients: a practical approach to diagnosis and management. Front Pediatr. 2019;7:133. 
16. Jia H, Thelwell C, Dilger P, Bird C, Daniels S, Wadhwa M. Endothelial cell functions impaired by interferon in vitro: insights into the molecular mechanism of thrombotic microangiopathy associated with interferon therapy. Thromb Res. 2018;163:105-16.

17. Jodele S, Davies SM, Lane A, Khoury J, Dandoy C, Goebel J, et al. Diagnostic and risk criteria for HSCT-associated thrombotic microangiopathy: a study in children and young adults. Blood. 2014;124: 645-53.

18. C. National High Blood Pressure Education Program Working Group on High Blood Pressure in, Adolescents. The fourth report on the diagnosis, evaluation, and treatment of high blood pressure in children and adolescents. Pediatrics. 2004;114:555-76.

19. Jodele S, Fukuda T, Mizuno K, Vinks AA, Laskin BL, Goebel J, et al. Variable eculizumab clearance requires pharmacodynamic monitoring to optimize therapy for thrombotic microangiopathy after hematopoietic stem cell transplantation. Biol Blood Marrow Transplant. 2016;22(2):307-15.

20. Dandoy C, Davies SM, Hirsch R, Chima RS, Paff Z, Cash M, et al. Abnormal echocardiography seven days after stem cell transplant may be an early indicator of thrombotic microangiopathy. Biol Blood Marrow Transplant. 2015;21(1):113-8.

21. Dandoy CE, Hirsch R, Chima R, Davies SM, Jodele S. Pulmonary hypertension after hematopoietic stem cell transplantation. Biol Blood Marrow Transplant. 2013;19:1546-56.

22. Warren M, Jodele S, Dandoy C, Myers KC, Wallace G, Nelson A, et al. A complete histologic approach to gastrointestinal biopsy from hematopoietic stem cell transplant patients with evidence of transplant-associated gastrointestinal thrombotic microangiopathy. Arch Pathol Lab Med. 2017;141:1558-66.

23. Pfeiffer TM, Rotz SJ, Ryan TD, Hirsch R, Taylor M, Chima R, et al. Pericardial effusion requiring surgical intervention after stem cell transplantation: a case series. Bone Marrow Transplant. 2017;52:630-3.

24. El-Bietar J, Warren M, Dandoy C, Myers KC, Lane A, Wallace G, et al. Histologic features of intestinal thrombotic microangiopathy in pediatric and young adult patients after hematopoietic stem cell transplantation. Biol Blood Marrow Transplant. 2015;21:19942001.

25. Vallurupalli M, Berliner N. Emapalumab for the treatment of relapsed/refractory hemophagocytic lymphohistiocytosis. Blood. 2019;134(21):1783-6.

26. Bae MN, Kwak DH, Park SJ, Choi BS, Park CW, Choi YJ, et al. Acute kidney injury induced by thrombotic microangiopathy in a patient with hemophagocytic lymphohistiocytosis. BMC Nephrol. 2016;17:4.

27. Allinovi M, Cirami CL, Caroti L, Antognoli G, Farsetti S, Amato $\mathrm{MP}$, et al. Thrombotic microangiopathy induced by interferon beta in patients with multiple sclerosis: three cases treated with eculizumab. Clin Kidney J. 2017;10:625-31.

28. Kundra A, Wang JC. Interferon induced thrombotic microangiopathy (TMA): analysis and concise review. Crit Rev Oncol Hematol. 2017;112:103-12.

29. Magro CM, Poe JC, Kim C, Shapiro L, Nuovo G, Crow MK, et al. Degos disease: a C5b-9/interferon-alpha-mediated endotheliopathy syndrome. Am J Clin Pathol. 2011;135:599-610.

30. Kavanagh D, McGlasson S, Jury A, Williams J, Scolding N, Bellamy C, et al. Type I interferon causes thrombotic microangiopathy by a dose-dependent toxic effect on the microvasculature. Blood. 2016;128:2824-33.

31. Alperin JM, Ortiz-Fernandez L, Sawalha AH. Monogenic lupus: a developing paradigm of disease. Front Immunol. 2018;9:2496.

32. Jodele S, Medvedovic M, Luebbering N, Chen J, Dandoy CE, Laskin BL, et al. Interferon-complement loop in transplantassociated thrombotic microangiopathy. Blood Adv. 2020;4: 1166-77.

33. Yuen J, Pluthero FG, Douda DN, Riedl M, Cherry A, Ulanova M, et al. NETosing neutrophils activate complement both on their own NETs and bacteria via alternative and non-alternative pathways. Front Immunol. 2016;7:137.

34. Cross AR, Glotz D, Mooney N. The role of the endothelium during antibody-mediated rejection: from victim to accomplice. Front Immunol. 2018;9:106.

35. Jodele S, Zhang K, Zou F, Laskin B, Dandoy CE, Myers KC, et al. The genetic fingerprint of susceptibility for transplant associated thrombotic microangiopathy. Blood. 2016;127(8):989-96.

36. Karapinar B, Yilmaz D, Balkan C, Akin M, Ay Y, Kvakli K. An unusual cause of multiple organ dysfunction syndrome in the pediatric intensive care unit: hemophagocytic lymphohistiocytosis. Pediatr Crit Care Med. 2009;10:285-90.

37. Dandoy CE, Linscott LL, Davies SM, Leach JL, Myers KC, ElBietar J, et al. Clinical utility of computed tomography and magnetic resonance imaging for diagnosis of posterior reversible encephalopathy syndrome after stem cell transplantation in children and adolescents. Biol Blood Marrow Transplant. 2015;21:2028-32.

38. Popeskou S, Gavillet M, Demartines N, Christoforidis D. Hemophagocytic lymphohistiocytosis and gastrointestinal bleeding: what a surgeon should know. Case Rep Surg. 2015;2015: 745848 .

Publisher's Note Springer Nature remains neutral with regard to jurisdictional claims in published maps and institutional affiliations. 